\title{
Line ratios for helium-like ions: Applications to collision-dominated plasmas ${ }^{\star}$
}

\author{
D. Porquet ${ }^{1,2}$, R. Mewe ${ }^{3}$, J. Dubau ${ }^{4,5}$, A. J. J. Raassen ${ }^{3,6}$, and J. S. Kaastra ${ }^{3}$
}

1 CEA/DSM/DAPNIA, Service d'Astrophysique, CEA Saclay, 91191 Gif-sur-Yvette Cedex, France

2 DAEC, Observatoire de Paris, Section Meudon, 92195 Meudon Cedex, France

3 Space Research Organization Netherlands (SRON), Sorbonnelaan 2, 3584 CA Utrecht, The Netherlands

4 LSAI, U.M.R. 8624, CNRS, Université de Paris Sud, 91405 Orsay Cedex, France

5 DARC, Observatoire de Paris, Section Meudon, 92195 Meudon Cedex, France

6 Astronomical Institute "Anton Pannekoek", Kruislaan 403, 1098 SJ Amsterdam, The Netherlands

Received 4 May 2001 / Accepted 21 June 2001

\begin{abstract}
The line ratios $R$ and $G$ of the three main lines of He-like ions (triplet: resonance, intercombination, forbidden lines) are calculated for $\mathrm{C}$ V, N VI, O VII, Ne IX, Mg XI, and Si XIII. These ratios can be used to derive electron density $n_{\mathrm{e}}$ and temperature $T_{\mathrm{e}}$ of hot late-type stellar coronae and O, B stars from high-resolution spectra obtained with Chandra (LETGS, HETGS) and XMM-Newton (RGS). All excitation and radiative processes between the levels and the effect of upper-level cascades from collisional electronic excitation and from dielectronic and radiative recombination have been considered. When possible the best experimental values for radiative transition probabilities are used. For the higher- $Z$ ions (i.e. NeIX, Mg XI, Si XIII) possible contributions from blended dielectronic satellite lines to each line of the triplets were included in the calculations of the line ratios $R$ and $G$ for four specific spectral resolutions: RGS, LETGS, HETGS-MEG, HETGS-HEG. The influence of an external stellar radiation field on the coupling of the $2^{3} \mathrm{~S}$ (upper level of the forbidden line) and $2^{3} \mathrm{P}$ levels (upper levels of the intercombination lines) is taken into account. This process is mainly important for the lower- $Z$ ions (i.e. $\mathrm{C} v, \mathrm{~N}$ VI, O viI) at moderate radiation temperature $\left(T_{\mathrm{rad}}\right)$. These improved calculations were done for plasmas in collisional ionization equilibrium, but will be later extended to photo-ionized plasmas and to transient ionization plasmas. The values for $R$ and $G$ are given in extensive tables, for a large range of parameters, which could be used directly to compare to the observations.
\end{abstract}

Key words. X-rays: stars - atomic processes - stars: coronae - stars: late-type - stars: activity - early-type stars: activity

\section{Introduction}

The new generation of X-ray satellites (Chandra, XMMNewton) enables us to obtain unprecedented high spectral resolution and high $S / N$ spectra. The wavelength

Send offprint requests to: D. Porquet, e-mail: dporquet@cea.fr

* Tables 4 to 69 and A.1 to A.6 are only available in electronic form at the CDS via anonymous ftp to cdsarc.u-strasbg.fr (130.79.128.5) or via http://cdsweb.u-strasbg.fr/cgi-bin/qcat?J/A+A/376/1113 ranges of the $R G S^{1}(6-35 \AA)$, of the $L E T G S^{2}(2-175 \AA)$, and of the $H E T G S^{3}$ (MEG range: 2.5-31 $\AA$; HEG range: 1.2-15 $\AA$ ) contain the helium-like line "triplets" from $\mathrm{CV}$ (or Nvi for the RGS, and for the HETGS-HEG) to Si XIII. The triplet consists of three close lines: the resonance line, the intercombination line and the forbidden line. The helium-like triplets provide electron density $\left(n_{\mathrm{e}} \sim 10^{8}-10^{13} \mathrm{~cm}^{-3}\right)$ as well as electron temperature

\footnotetext{
1 RGS: Reflection Grating Spectrometer on board XMMNewton (den Herder et al. 2001).

2 LETGS: Low Energy Transmission Grating Spectrometer on board Chandra (Brinkman et al. 2000).

3 HETGS: High Energy Transmission Grating Spectrometer on board Chandra (Canizares et al. 2000).
} 
$(T \sim 1-10 \mathrm{MK})$ as first shown by Gabriel \& Jordan (1969). The line ratios of these He-like triplets enable also the determination of the ionization processes (photoionization and/or collisional ionization) which prevail in the plasma (Porquet \& Dubau 2000; Liedahl 1999).

The ratios of these lines are already widely used for collisional solar plasma diagnostics (e.g., Gabriel \& Jordan 1969; Doyle 1980; Keenan et al. 1987; McKenzie \& Landecker 1982).

Recently, also theoretical calculations for photoionized plasmas or "hybrid" plasmas (photo-ionization plus collisional ionization) have been given by Porquet \& Dubau (2000) (hereafter referred to as Paper I). Their calculations have been already applied to spectra of Seyfert galaxies (e.g. NGC 5548, Kaastra et al. 2000; Mkn 3, Sako et al. 2000; NGC 4151, Ogle et al. 2000; NGC 4051, Collinge et al. 2001, etc.).

We present here calculations of these ratios, from $\mathrm{CV}$ to Si XIII, which could be applied directly for the first time to Chandra and XMM-Newton observations of extra-solar collisional plasmas such as stellar coronae. These calculations have been done to apply an improved model to the density analysis of the RGS, the LETGS and the $H E T G S$ spectra of various late-type stars such as Capella, Procyon, and $\alpha$ Centauri (e.g., Audard et al. 2001; Ness et al. 2001a; Mewe et al. 2001) and also to O stars such as $\zeta$ Puppis (Kahn et al. 2001). Our model is to be considered as an improvement of various previous calculations for solar plasmas such as done by e.g., Gabriel \& Jordan (1969), Blumenthal et al. (1972), Mewe (1972), Mewe \& Schrijver (1978a, 1978b, 1978c), Pradhan \& Shull (1981), Mewe \& Gronenschild (1981), Mewe et al. (1985), and Pradhan $(1982,1985)$. The calculations are partly based on recent work by Porquet \& Dubau (2000).

In the next three sections, we introduce the plasma diagnostics and the atomic processes and atomic data taken into account in the calculations. In Sect. 5, we display the results for $R$ and $G$ calculated for four specific spectral resolutions (Full Width at Half Maximum: $F W H M$ ): RGS, LETGS, HETGS-MEG, HETGS-HEG, over a broad range of physical parameters: $n_{\mathrm{e}}, T_{\mathrm{e}}$, radiation temperature $\left(T_{\text {rad }}\right)$, and radiation dilution factor $(W)$.

\section{Plasma diagnostics}

In the X-ray range, the three most intense lines of heliumlike ions ("triplet") are: the resonance line ( $w$, also called $\left.r: 1 \mathrm{~s}^{2}{ }^{1} \mathrm{~S}_{0}-1 \mathrm{~s} 2 \mathrm{p}^{1} \mathrm{P}_{1}\right)$, the intercombination lines $(x+y$, also called $\left.i: 1 \mathrm{~s}^{2}{ }^{1} \mathrm{~S}_{0}-1 \mathrm{~s} 2 \mathrm{p}^{3} \mathrm{P}_{2,1}\right)$ and the forbidden line $(z$, also called $\left.f: 1 \mathrm{~s}^{2}{ }^{1} \mathrm{~S}_{0}-1 \mathrm{~s} 2 \mathrm{~s}{ }^{3} \mathrm{~S}_{1}\right)$. They correspond to transitions between the $n=2$ shell and the $n=1$ ground-state shell (see Fig. 1). The wavelengths in $\AA$ of each line from $\mathrm{CV}$ $(Z=6)$ to $\operatorname{Si~XIII~}(Z=14)$ are reported in Table 1.

As shown by Gabriel \& Jordan (1969), the ratios defined below are sensitive to the electron density and to the

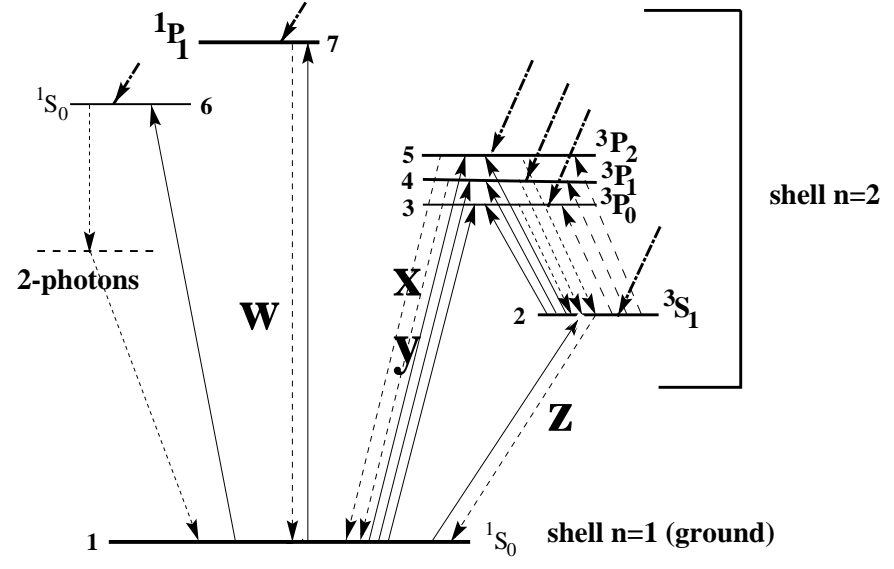

Fig. 1. Simplified level scheme for helium-like ions. $w$ (or $\mathrm{r}$ ), $x, y$ (or i), and $z$ (or $\mathrm{f}$ ): resonance, intercombination, and forbidden lines, respectively. Full upward arrows: collisional excitation transitions, broken arrows: radiative transitions (including photo-excitation from $2{ }^{3} \mathrm{~S}_{1}$ to $2{ }^{3} \mathrm{P}_{0,1,2}$ levels, and 2-photon continuum from $2{ }^{1} \mathrm{~S}_{0}$ to the ground level), and thick skew arrows: recombination (radiative and dielectronic) plus cascade processes.

Table 1. Wavelengths in $\AA$ of the three main X-ray lines of Cv, Nvi, O viI, NeIx, MgXI and SixiII (from Vainshtein \& Safronova 1978).

\begin{tabular}{cccccccc}
\hline \hline line & label & $\mathrm{C}$ V & N VI & O VII & NeIX & Mg XI & Si XIII \\
\hline resonance & $w(r)$ & 40.279 & 28.792 & 21.603 & 13.447 & 9.1681 & 6.6471 \\
inter- & $x$ & 40.711 & 29.074 & 21.796 & 13.548 & 9.2267 & 6.6838 \\
combination & $y$ & 40.714 & 29.076 & 21.799 & 13.551 & 9.2298 & 6.6869 \\
forbidden & $z(f)$ & 41.464 & 29.531 & 22.095 & 13.697 & 9.3134 & 6.7394 \\
\hline \hline
\end{tabular}

electron temperature:

$R\left(n_{\mathrm{e}}\right)=\frac{z}{x+y} \quad\left(\right.$ also $\left.\frac{f}{i}\right)$
$G\left(T_{\mathrm{e}}\right)=\frac{z+(x+y)}{w} \quad\left(\right.$ also $\left.\frac{f+i}{r}\right)$.

\subsection{Density diagnostic}

In the low-density limit, all $n=2$ states are populated directly or via upper-level radiative cascades by electron impact from the He-like ground state and/or by (radiative and dielectronic) recombination of $\mathrm{H}$-like ions (see Fig. 2). These states decay radiatively directly or by cascades to the ground level. The relative intensities of the three intense lines are then independent of density. As $n_{\mathrm{e}}$ increases from the low-density limit, some of these states $\left(1 \mathrm{~s} 2 \mathrm{~s}^{3} \mathrm{~S}_{1}\right.$ and $\left.{ }^{1} \mathrm{~S}_{0}\right)$ are depleted by collisions to the nearby 
states where $n_{\text {crit }} \mathrm{C} \sim \mathrm{A}$, with $\mathrm{C}$ being the collisional coefficient rate, A being the radiative transition probability from $n=2$ to $n=1$ (ground state), and $n_{\text {crit }}$ being the critical density. Collisional excitation depopulates first the $1 \mathrm{~s} 2 \mathrm{~s}^{3} \mathrm{~S}_{1}$ level (upper level of the forbidden line) to the $1 \mathrm{~s} 2 \mathrm{p}^{3} \mathrm{P}_{0,1,2}$ levels (upper levels of the intercombination lines). The intensity of the forbidden line decreases while those of the intercombination lines increase, hence implying a reduction of the ratio $R$ (according to Eq. (1)), over approximately two or three decades of density (see Fig. 8 in Paper I). For much higher densities, $1 \mathrm{~s} 2 \mathrm{~s}{ }^{1} \mathrm{~S}_{0}$ is also depopulated to $1 \mathrm{~s} 2 \mathrm{p}^{1} \mathrm{P}_{1}$, and the resonance line becomes sensitive to the density (this has been nicely illustrated by Gabriel \& Jordan 1972 in their Fig. 4.6.9).

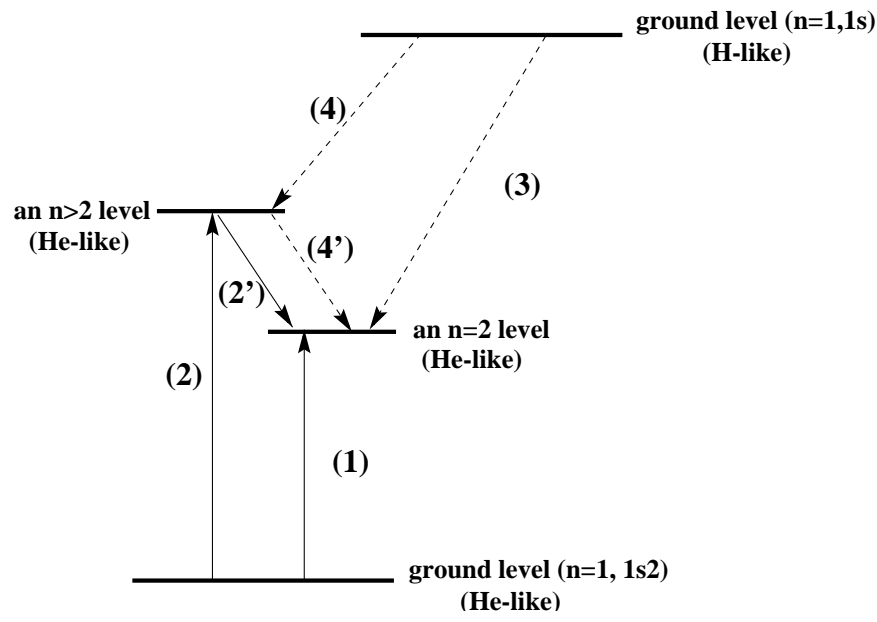

Fig. 2. Simplified diagram showing the different contributions to the population of a given $n=2$ shell level. (1): direct contribution due to collisional excitation from the ground level $\left(1 \mathrm{~s}^{2}\right)$ of He-like ions; $(2)+\left(2^{\prime}\right)$ : collisional excitation plus upper-level radiative cascade contribution; (3): direct radiative recombination or direct dielectronic recombination from H-like ions; and $(4)+\left(4^{\prime}\right)$ : radiative recombination or dielectronic recombination plus upper-level radiative cascade contribution.

However, caution should be applied for low- $Z$ ions (i.e. $\mathrm{C}$ V, N VI, O VII) since in the case of an intense UV radiation field, the photo-excitation between the ${ }^{3} \mathrm{~S}$ term and the ${ }^{3} \mathrm{P}$ term is not negligible. This process has the same effect on the forbidden line and on the intercombination line as the collisional coupling, i.e. lowering of the ratio $R$, and thus could mimic a high-density plasma. It should be taken into account to avoid any confusion between a highdensity plasma and a high radiation field (see Sect. 4.4 for more details).

\subsection{Temperature and ionization process diagnostics}

The ratio $G$ (see Eq. (2)) is sensitive to the electron temperature since the collisional excitation rates do not have the same dependence on temperature for the resonance line as for the forbidden and intercombination lines.

In addition, as detailed in Paper I (see also Mewe 1999; Liedahl 1999), the relative intensity of the resonance $w$ (or $r$ ) line, compared to the forbidden $z$ (or $f$ ) and the intercombination $x+y$ (or $i$ ) lines, contains information about the ionization processes that occur: a strong resonance line compared to the forbidden or the intercombination lines corresponds to collision-dominated plasmas. It leads to a ratio of $G=(z+(x+y)) / w \sim 1$ (or $(f+i) / r \sim 1)$. On the contrary, a weak resonance line corresponds to plasmas dominated by photo-ionization $(G=(z+(x+y)) / w>4$, or $(f+i) / r>4)$.

\section{Schematic model}

We illustrate the relevant processes in the formation of the resonance, intercombination, and forbidden lines with a simplified level scheme (cf. Mewe \& Schrijver 1978a) consisting of the following levels denoted by short labels: g: ground level $1^{1} \mathrm{~S}_{0} ; m^{\prime}$ : upper metastable level $2^{1} \mathrm{~S}_{0}$ of the two-photon transition; $1^{\prime}$ : upper level $2^{1} \mathrm{P}_{1}$ of the resonance line; $m$ : upper metastable level $2^{3} \mathrm{~S}_{1}$ of the forbidden ( $f)$ line; $\mathrm{p}_{k}\left(k=1,2,3\right.$ : levels $2^{3} \mathrm{P}_{k}\left(2^{3} \mathrm{P}_{1}\right.$ is the upper level of the by far the strongest component $(y)$ of the intercombination line $i$, and $2{ }^{3} \mathrm{P}_{2}$ is the upper level of the weaker component $(x)$ ); c: continuum level which lumps together all higher levels to represent the cascades from excitation and recombination processes.

The electron collisional rate coefficient (in $\mathrm{cm}^{3} \mathrm{~s}^{-1}$ ) for transition $j \rightarrow k$ is written as:

$C_{j k}=8.63 \times 10^{-6} \frac{\gamma_{j k}}{w_{j} \sqrt{T_{\mathrm{e}}}} \exp \left(-\frac{\Delta E_{j k}}{k T_{\mathrm{e}}}\right)$,

where $\Delta E_{j k}$ is the excitation energy, $T_{\mathrm{e}}$ is the electron temperature in $\mathrm{K}, \gamma_{j k}$ the collision strength averaged over a Maxwellian electron energy distribution, and $w_{j}$ the statistical weight of the initial level $j$.

The total rate coefficients for the formation of the forbidden ( $f$ or $z$ notation) and intercombination $(i$ or $x+y$ ) line can be written as (e.g., Mewe \& Schrijver 1978a, Eqs. (18-30)):

$$
\begin{aligned}
I_{f} & =B R_{m g}\left[C_{g m}+\sum_{k=0}^{2} B R_{p_{k} m} C_{g p_{k}}\right], \\
I_{i} & =\sum_{k=1}^{2} B R_{p_{k} g}\left[C_{g p_{k}}+B R_{m p_{k}} C_{g m}\right],
\end{aligned}
$$

with the various branching ratios:

$$
\begin{gathered}
B R_{m g}=\frac{A_{m g}}{A_{m g}+n_{\mathrm{e}} S_{m p}^{\prime}} \\
S_{m p}^{\prime}=\sum_{k=1}^{2} C_{m p_{k}} B R_{p_{k} g}^{\prime}
\end{gathered}
$$




$$
\begin{aligned}
& B R_{p_{k} m}=\frac{A_{p_{k} m}}{A_{p_{k} m}+A_{p_{k} g}}\left(B R_{p_{0} m} \equiv 1\right), \\
& B R_{p_{k} g}^{\prime}=\frac{A_{p_{k} g}}{A_{p_{k} g}+A_{p_{k} m}}\left(B R_{p_{0} g}^{\prime} \equiv 0\right), \\
& B R_{p_{k} g}=\frac{A_{p_{k} g}}{A_{p_{k} g}+A_{p_{k} m} B R_{m g}}\left(B R_{p_{0} g} \equiv 0\right), \\
& B R_{m g}=\frac{A_{m g}}{A_{m g}+n_{\mathrm{e}} S_{m p}}, \\
& B R_{m p_{k}}=\frac{n_{\mathrm{e}} C_{m p_{k}}}{A_{m g}+n_{\mathrm{e}} S_{m p}}, \\
& S_{m p}=\sum_{k=0}^{2} C_{m p_{k}} .
\end{aligned}
$$

Analogously, we can derive the total rate coefficients for the formation of the resonance ( $r$ or $w$ ) line or two-photon radiation $(2 \mathrm{ph})$ by substituting $m \rightarrow m^{\prime}, p, p_{k} \rightarrow 1^{\prime}$ and performing no summation:

$$
\begin{aligned}
& I_{r}=B R_{1^{\prime} g}\left[C_{g 1^{\prime}}+B R_{m^{\prime} 1^{\prime}} C_{g m^{\prime}}\right], \\
& I_{2 p h}=B R_{m^{\prime} g}\left[C_{g m^{\prime}}+B R_{1^{\prime} m} C_{g 1^{\prime}}\right],
\end{aligned}
$$

and changing all $B R$ 's etc. appropriately.

We note that the collision coefficients $C_{j k}$ include also the $n>2$ cascades. The radiative transition probabilities are tabulated in the Table 2 of Paper I and the effective collision strengths $\gamma$ in their Tables $9-13$ and in their Fig. 4. We assume in this section that the electron density is so low that the collision de-excitation rate $n_{\mathrm{e}} C_{p_{k} m}$ can be neglected with respect to the spontaneous radiative rate $A_{p_{k} m}$ (e.g., for $\mathrm{C} \mathrm{V} n_{\mathrm{e}} C_{p_{k} m}<A_{p_{k} m}$ for $\left.n_{\mathrm{e}}<10^{15} \mathrm{~cm}^{-3}\right)$. This can be easily taken into account by the substitution

$$
A_{p_{k} m} \rightarrow A_{p_{k} m}+n_{\mathrm{e}} C_{p_{k} m}
$$

Further, all collision processes that couple the singlet and triplet system are neglected in this schematic model. It turns out that at high density the coupling $m, p_{k} \leftrightarrow m^{\prime}, 1^{\prime}$ cannot be neglected.

In the full calculations used in this work (see Sect. 5), the coupling between the singlet and triplet system has been taken into account, as well as the collisional deexcitation.

If we take also into account the contribution from radiative and dielectronic recombinations of the hydrogenlike ion we substitute

$C_{g k} \rightarrow C_{g k}+\alpha_{c k}^{\prime}$

where $k=m$ or $k=p_{k}$, respectively, and

$\alpha_{c k}^{\prime}=\left(N_{\mathrm{H}} / N_{\mathrm{He}}\right) \alpha_{c k}$,

where $\alpha_{c k}$ is the total radiative and dielectronic recombination rate coefficient including cascades and $N_{\mathrm{H}} / N_{\mathrm{He}}$ is the abundance ratio of hydrogen-like to helium-like ions (e.g. taken from Arnaud \& Rothenflug 1985; Mazzotta et al. 1998). The recombination coefficients are given in Tables 3-8 in Paper I. However, for a collision-dominated plasma, the recombination generally gives only a minor effect (i.e. far less than few percents), but are nevertheless introduced in the full calculations in Sect. 5 .

Mewe \& Schrijver (1978a) took also into account the effect of a stellar radiation field (also called photoexcitation) with effective radiation temperature $T_{\text {rad }}$. This can be done by substituting in the above equations:

$n_{\mathrm{e}} C_{m p_{k}} \rightarrow n_{\mathrm{e}} C_{m p_{k}}+B_{m p_{k}}$

$n_{\mathrm{e}} S_{m p} \rightarrow n_{\mathrm{e}} S_{m p}+B_{m p}$

where $B_{m p}=\sum_{k=0}^{2} B_{m p_{k}}$ and

$B_{m p_{k}}=\frac{W A_{p_{k} m}\left(w_{p_{k}} / w_{m}\right)}{\exp \left(\frac{\Delta E_{m p_{k}}}{k T_{\mathrm{rad}}}\right)-1}$

is the rate (in $\mathrm{s}^{-1}$ ) of absorption $m \rightarrow p_{k}$ and $W$ is the dilution factor of the radiation field (as a special case we take $W=\frac{1}{2}$ close to the stellar surface). We have checked that the radiation field is so low that the induced emission rate $B_{p_{k} m}=\left(w_{m} / w_{p_{k}}\right) B_{m p_{k}}$ is negligible respect to the spontaneous radiative rate $A_{p_{k} m}$. Nevertheless, in the full calculations used in this work (see Sect. 5), we have taken into account induced emission as well as photo-excitation.

Mewe \& Schrijver (1978a) considered also inner-shell ionization of the Lithium-like ion which can give an important contribution to the forbidden line in a transient plasma (Mewe \& Schrijver 1978b) such as a supernova remnant. However, in the present calculations we neglect this because we consider plasmas in ionization equilibrium.

Finally, Mewe \& Schrijver (1978a, 1978c) have considered also excitation $2^{3} \mathrm{~S} \rightarrow 2^{3} \mathrm{P}$ by proton collisions using approximations of Coulomb-Born results from Blaha (1971). In a new version (SPEX90) of our spectral code SPEX (Kaastra et al. 1996), which contains for the $\mathrm{H}$ and He-like ions an improvement of the known MEKAL code (Mewe et al. 1985, 1995a), proton collisions are taken into account based on Blaha's results. Test calculations with SPEX90 show that for an equilibrium plasma in all practical cases proton excitation is negligible compared to electron excitation.

In the case where $N_{\mathrm{H}} / N_{\mathrm{He}} \gg 1$, recombination is dominant e.g., for photo-ionized plasmas it turns out that the ratios $R \equiv f / i$ are for collisional and photo-ionized plasmas comparable in the same density range (cf. also Mewe $1999)$, but the ratios $G \equiv(i+f) / r$ are very different, e.g., $G \sim 1$ for a collisional plasma and a factor $\sim 2-4$ larger for a photo-ionized plasma where the resonance line is relatively much weaker. 


\section{Atomic data and improvements}

The intensities of the three lines (resonance, forbidden and intercombination) are calculated mainly from atomic data presented in Paper I (Porquet \& Dubau 2000). In this work (as well as in Paper I) for all temperatures (low and high), radiative recombination contributions (direct + upperlevel radiative cascades), and collisional excitations inside the $n=2$ shell were included in the line ratio calculations. For high temperatures, the collisional excitation contribution (direct + near-threshold resonance + cascades $)$ from the ground level ( $n=1$ shell, $1 \mathrm{~s}^{2}$ ) are important as well as dielectronic recombination (direct + cascades).

Excitation collisional data are also taken from Paper I, which are based on the calculations from Zhang \& Sampson (1987) plus the contribution of the upper-level $(n>2)$ radiative cascades calculated in Paper I (see Paper I for more details).

The ionization balance is from Mazzotta et al. (1998) and the data for radiative and dielectronic recombinations are from Paper I. Various new data for the transition probabilities (e.g., forbidden and intercombination lines) have been selected (see Sect. 4.1).

In the following paragraphs we describe the several differences between Paper I and this work: $A_{k i}$, optical depth, contribution of the blended dielectronic satellite lines, and radiation field.

\subsection{Update of the $A_{k i}$ for the forbidden and the intercombination lines}

We have updated the transition probabilities $A_{k i}$ reported in Paper I for the intercombination $\left(y, A_{1 \rightarrow 4}\right)$ and the forbidden $\left(z, A_{1 \rightarrow 2}\right)$ line by published experimental values (see Table 2, and references therein). In some cases, no published experimental values have been found and then we used the theoretical values from Lin et al. (1977). Indeed, comparisons of their theoretical values with the experimental values reported in Table 2 seem to show good agreement in other cases.

For $\mathrm{CV}$, the ratio $R$ is reduced by about $20 \%$ comparing the calculations using the values of $A_{k i}$ reported in Paper I, while for N vi the reduction is less than $10 \%$. For O VII, NeIX, MgXI, and Si XIII, the differences between the current calculations using these new values of $A_{k i}$ and those reported in Paper I are negligible.

\subsection{Influence of the optical depth (resonant scattering)}

Schrijver et al. (1995) and Mewe et al. (1995b) have investigated the possibility that resonance photons are scattered out of the line of sight in late-type stellar coronae (see also Acton 1978). Indeed, in this process, a resonance
Table 2. Update of the transition probabilities $\left(\mathrm{s}^{-1}\right)$ with published experimental values for the forbidden line $\left(z, A_{1 \rightarrow 2}\right)$ and the intercombination line $\left(y, A_{1 \rightarrow 4}\right)$ compared to the theoretical values from Porquet \& Dubau (2000).

\begin{tabular}{lll}
\hline \hline ion & $\begin{array}{l}\text { forbidden line } \\
\left(z, A_{1 \rightarrow 2}\right)\end{array}$ & $\begin{array}{l}\text { intercombination line } \\
\left(y, A_{1 \rightarrow 4}\right)\end{array}$ \\
\hline $\mathrm{C}_{\mathrm{V}}$ & $4.857(+1)(\mathrm{S} 94)$ & $2.90(+7)(\mathrm{H} 85)$ \\
$\mathrm{N}$ VI & $2.538(+2)(\mathrm{N} 00)$ & $1.38(+8)(\mathrm{H} 85)$ \\
$\mathrm{O}_{\text {VII }}$ & $1.046(+3)(\mathrm{C} 98)$ & $5.800(+8)(\mathrm{E} 81)$ \\
$\mathrm{Ne} \mathrm{IX}$ & $1.09(+4)(\mathrm{T} 99)$ & $5.400(+9) *$ \\
$\mathrm{Mg}$ XI & $7.347(+4)(\mathrm{S} 95)$ & $3.448(+10)(\mathrm{A} 81)$ \\
$\mathrm{Si} \mathrm{XIII}$ & $3.610(+5)^{*}$ & $1.497(+11)(\mathrm{A} 79)$ \\
\hline \hline
\end{tabular}

(A79): Armour et al. (1979)

(A81): Armour et al. (1981)

(C98): Crespo López-Urrutia et al. (1998)

(E81): Engström et al. (1981)

(H85): Hutton et al. (1985)

(N00): Neill et al. (2000)

(S94): Schmidt et al. (1994)

(S95): Stefanelli et al. (1995)

(T99): Träbert et al. (1999)

*: Theoretical values taken from Lin et al. (1977), see text (Sect. 4.1).

line photon is absorbed by an ion in the ground state and then re-emitted, generally in a different direction. So, the total photon intensity integrated over $4 \pi$ remains unchanged but the photon distribution with respect to a given direction is altered. This absorption and re-emission is indistinguishable from scattering and depends on the geometry of the region being observed. In general, photons would be scattered preferentially out of the line of sight for active regions (relatively dense areas) and into the line of sight for the surrounding quiet Sun (less dense area), see Schmelz et al. (1997) and Mewe et al. (2001). The effect is smaller for instruments with a larger field of view.

This could have an impact on the temperature diagnostic, the so-called $G=(z+x+y) / w$ or $(f+i) / r$ ratio. If the optical depth of the line is not taken into account, the calculated intensity ratio $G$ can be overestimated and the inferred temperature from the $G$ ratio is underestimated.

As detailed in Mewe et al. (2001), branching ratios can be used to check the assumption of the optical thin model because effects of resonance scattering would affect the measured branching ratio. From the fact that the intensities of e.g., the strong resonance lines FexviII $\lambda 93.92$ and Fe XIX $\lambda 108.307$ are in good agreement with the intensities of other lines sharing the same upper level, one can derive a constraint on the optical depth taking into account the systematic uncertainties of the theoretical transition probabilities A (typical 25\% for each A, hence 35\% for the branching ratio) which dominate over the statistical 
errors (typically 10\%). If we rule out a reduction in the resonance line intensity larger than about $30 \%$, then on the basis of a simple "escape-factor" model with

$P(\tau) \simeq \frac{1}{[1+0.43 \tau]}$,

the escape factor for a homogeneous mixture of emitters and absorbers in a slab geometry (e.g., Kaastra \& Mewe 1995), one can put a constraint on the optical depth. The optical depth $\tau$ for a Doppler-broadened resonance line can be written as (Mewe et al. 1995b) ${ }^{4}$ :

$$
\begin{aligned}
\tau & =1.16 \times 10^{-17}\left(\frac{n_{i}}{n_{e l}}\right) A_{Z}\left(\frac{N_{\mathrm{H}}}{n_{\mathrm{e}}}\right) \lambda f \sqrt{\frac{M}{T_{\mathrm{e}}}} n_{\mathrm{e}} l \\
& \equiv 10^{-19} C_{d}\left(\frac{A_{Z}}{A_{Z_{\odot}}}\right)\left(\frac{n_{\mathrm{e}} l}{\sqrt{T_{6}}}\right)
\end{aligned}
$$

where $\left(n_{i} / n_{e l}\right)$ is the ion fraction (e.g. from Arnaud \& Rothenflug 1985, from Arnaud \& Raymond 1992 for iron, or from Mazzotta et al. 1998), $A_{Z}$ is the elemental abundance relative to hydrogen, $A_{Z_{\odot}}$ the corresponding value for the solar photosphere as given by Anders \& Grevesse (1989), $N_{\mathrm{H}} / n_{\mathrm{e}} \simeq 0.85$ the ratio of hydrogen to electron density, $\lambda$ is the wavelength in $\AA, f$ the absorption oscillator strength, $M$ is the atomic weight, $T_{\mathrm{e}}$ is the electron temperature (in $\mathrm{K}$ or $T_{6}$ in $\mathrm{MK}$ ), $l$ a characteristic plasma dimension (in $\mathrm{cm}$ ) and

$C_{\mathrm{d}} \equiv 98.5\left(\frac{n_{i}}{n_{e l}}\right) A_{Z_{\odot}} \lambda f \sqrt{M}$.

According to Eq. (23), Ness et al. (2001a) estimated the optical depth, adopting a value of unity for the fractional ionization and using solar abundances. One further assumes $T_{\mathrm{e}}$ at the peak line formation, but note that $\tau$ is rather insensitive to the precise value of $T_{\mathrm{e}}$. One can determine - for each resonance line - that value of $n_{\mathrm{e}} \ell$ which yields an optical depth of unity. According to the values of $n_{\mathrm{e}}$ inferred from the ratio $R=z /(x+y)$ or $R=f / i$ (from $\mathrm{CV}$ to SixIII the intercombination and the forbidden lines are not sensitive to resonant scattering below a column density of $\mathcal{N}_{\mathrm{H}} \sim 10^{25-26} \mathrm{~cm}^{-2}$ and $\mathcal{N}_{\mathrm{H}} \sim 10^{30-31} \mathrm{~cm}^{-2}$, respectively, while the resonance line becomes sensitive to the resonant scattering above $\mathcal{N}_{\mathrm{H}} \sim 10^{21-23} \mathrm{~cm}^{-2}$ ), one can determine the corresponding values of $\ell$. One can compute the respective emission measures of $n_{\mathrm{e}}^{2} \ell^{3}$ respectively, and can compare these emission measures with those derived from the measured line fluxes $f_{\lambda}$ according to

$$
E M=\frac{4 \pi d^{2} f_{\lambda}}{P_{\lambda}\left(T_{\mathrm{e}}\right)}
$$

with the line emissivity $P_{\lambda}\left(T_{\mathrm{e}}\right)$ and the distance $d$ of the star. If the former is larger, this inconsistency shows that the assumption of a non-negligible optical depth is invalid

\footnotetext{
${ }^{4}$ The forefactor in the first part of Eq. (23) was in Mewe et al. $(1995 \mathrm{~b}, 2001)$ erroneously taken a factor of $10^{3}$ too large, but the 2nd part of Eq. (23) is still correct.
}

and we conclude that optical depth effects are irrelevant for the analysis of He-like triplets. On the contrary, the effect of resonant scattering should be taken into account when comparing the theoretical values with the observational ones.

Since $\tau_{\mathrm{r}}>>\tau_{\mathrm{i}}\left(\tau_{\mathrm{r}}\right.$ and $\tau_{\mathrm{i}}$ corresponding respectively to the optical depth of the resonance and the intercombination lines), we can write $G_{\tau} \equiv \frac{G}{P_{r}}$, where $G_{\tau}$ is the value of the ratio taken into account the optical depth of the resonance line, $G$ is the value without resonant scattering (such as in Paper I and Sect. 5), and $P_{\mathrm{r}}$ is the escape probability for the resonance line (Eq. (22)). One should note that $G_{\tau}$ is not strictly exact when the contribution of the blended dielectronic satellite lines are introduced in the calculations (see Sect. 4.3).

\subsection{Blended dielectronic satellite lines}

The intensity of a dielectronic satellite line arising from a doubly excited state with principal quantum number $n$ in a Lithium-like ion produced by dielectronic recombination of a He-like ion is given by:

$I_{\mathrm{s}}=N_{\mathrm{He}} n_{\mathrm{e}} C_{\mathrm{s}}$,

where $N_{\mathrm{He}}$ is the population density of the considered Helike ion in the ground state $1 \mathrm{~s}^{2}$ with statistical weight $g_{1}$ (for He-like ions $g_{1}=1$ ).

The rate coefficient (in $\mathrm{cm}^{3} \mathrm{~s}^{-1}$ ) for dielectronic recombination is given by (Bely-Dubau et al. 1979):

$C_{\mathrm{s}}=2.0706 \times 10^{-16} \frac{\mathrm{e}^{-E_{\mathrm{s}} / k T_{\mathrm{e}}}}{g_{1} T_{\mathrm{e}}^{3 / 2}} F_{2}(s)$,

where $E_{\mathrm{S}}$ is the energy of the satellite level $s$ with statistical weight $g_{\mathrm{s}}$ above the ground state of the He-like ion, $T_{\mathrm{e}}$ is the electron temperature in $\mathrm{K}$, and $F_{2}(s)$ is the so-called line strength factor (often of the order of about $10^{13} \mathrm{~s}^{-1}$ for the stronger lines) given by

$F_{2}(s)=\frac{g_{\mathrm{s}} A_{\mathrm{a}} A_{\mathrm{r}}}{\left(A_{\mathrm{a}}+\sum A_{\mathrm{r}}\right)}$,

where $A_{\mathrm{a}}$ and $A_{\mathrm{r}}$ are transition probabilities $\left(\mathrm{s}^{-1}\right)$ by autoionization and radiation, and the summation is over all possible radiative transitions from the satellite level $s$.

For a group of satellites with the same principal quantum number $n, E_{\mathrm{s}}$ can be approximated by

$E_{\mathrm{s}}[\mathrm{eV}]=1.239842 \times 10^{4} \frac{\mathrm{a}_{\mathrm{DR}}}{\lambda}$,

where $\lambda$ is the wavelength $(\AA)$ of the satellite line and $a_{\mathrm{DR}} \simeq 0.7,0.86,0.92$, and 0.96 for $n=2,3,4$, and $>4$, respectively (Mewe \& Gronenschild 1981). For $\lambda$ in $\AA$ and $T$ in $\mathrm{K}$ we can write:

$\frac{E_{\mathrm{s}}}{k T}=\frac{a_{\mathrm{DR}} h c}{\lambda k T}=1.439 \times 10^{8} \frac{a_{\mathrm{DR}}}{\lambda T}$. 
The influence of the blending of dielectronic satellite lines for the resonance, the intercombination and the forbidden lines has been taken into account where their contribution is not negligible in the calculation of $R$ and $G$, affecting the inferred electron temperature and density. This is the case for the high- $Z$ ions, i.e. Ne IX, Mg XI, and Si XIII $(Z=10,12$, and 14 , respectively). Since the contribution of the blended dielectronic satellite lines depends on the spectral resolution considered, we have estimated the ratios $R$ and $G$ for four specific spectral resolutions $(F W H M)$ : RGS-1 at the first order (i.e. $\Delta \lambda=0.073$, 0.075 and $0.078 \AA$ for NeIX, MgXI and SixIII respectively), LETGS (i.e. $\Delta \lambda=0.05 \AA$ ), HETGS-MEG (i.e. $\Delta \lambda=0.023 \AA$ ), and HETGS-HEG (i.e. $\Delta \lambda=0.012 \AA$ ).

For the $n=2,3,4$ blended dielectronic satellite lines we use the atomic data reported in the Appendix. For the higher- $n$ blended dielectronic satellite lines we use the results from Karim and co-workers. For $Z=10$ (Ne IX) we use the data from Karim (1993) who gives the intensity factor $F_{2}^{*} \equiv F_{2} / g_{1}$ for the strongest $\left(F_{2}^{*}>10^{12} \mathrm{~s}^{-1}\right)$ dielectronic satellite lines with $n=5-8$. For $Z=14$ (Si XIII), we take the calculations from Karim \& Bhalla (1992) who report the intensity factor $F_{2}^{*}$ for the strongest $\left(F_{2}^{*}>10^{12} \mathrm{~s}^{-1}\right)$ dielectronic satellite lines with $n=5-8$. For $Z=12$ (Mg XI) we have interpolated between the calculations from Karim (1993) for $Z=10$, and from Karim \& Bhalla (1992) for $Z=14$.

Including the contribution of the blended dielectronic satellite lines, we write for the ratios $R$ and $G$ :

$$
\begin{aligned}
& R=\frac{z+\text { sat } z}{(x+y)+\text { satxy }} \\
& G=\frac{(z+\text { sat } z)+((x+y)+\text { satxy })}{(w+\text { satw })},
\end{aligned}
$$

where satz, satxy and satz are respectively the contribution of blended dielectronic satellite lines to the forbidden line, to the intercombination lines, and to the resonance line, respectively. One can note that at very high density the ${ }^{3} \mathrm{P}$ levels are depleted to the ${ }^{1} \mathrm{P}$ level, and in that case $x+y$ decreases and $R$ tends to satz/satxy.

At the temperature at which the ion fraction is maximum for the He-like ion (see e.g. Arnaud \& Rothenflug 1985; Mazzotta et al. 1998), the differences between the calculations for $R$ (for $G$ ) with or without taking into account the blended dielectronic satellite lines are only of about $1 \%(9 \%), 2 \%(5 \%)$, and $5 \%(3 \%)$ for Ne IX, Mg XI, and Si XIII at the low-density limit and for $T_{\mathrm{rad}}=0 \mathrm{~K}$, respectively. On the other hand, for much lower electron temperatures, the effect is bigger since the intensity of the dielectronic satellite lines is proportional to $T_{\mathrm{e}}^{-3 / 2}$. As well, for high values of density $\left(n_{\mathrm{e}}\right)$ at which the intensity of the forbidden line is very weak (i.e. tends to zero), the contribution of the blended dielectronic satellite lines to the forbidden line leads to a ratio $R$ which decreases much slower with $n_{\mathrm{e}}$ than in the case where the contribution of the blended dielectronic satellite lines is not taken into account.

\subsection{Influence of a radiation field}

Recently, Kahn et al. (2001) have found with the $R G S$ on XMM-Newton that for $\zeta$ Puppis, the forbidden to intercombination line ratios within the helium-like triplets are abnormally low for N VI, O viI, and Ne IX. While this is sometimes indicative of a high electron density, they have shown that in the case of $\zeta$ Puppis, it is instead caused by the intense radiation field of this star. This constrains the location of the X-ray emitting shocks relative to the star, since the emitting regions should be close enough to the star in order that the UV radiation is not diluted too much.

A strong radiation field can mimic a high density if the upper $\left({ }^{3} \mathrm{~S}\right)$ level of the forbidden line is significantly depopulated via photo-excitation to the upper $\left({ }^{3} \mathrm{P}\right)$ levels of the intercombination lines, analogously to the effect of electronic collisional excitation (Fig. 1). The result is an increase of the intercombination lines and a decrease of the forbidden line.

Equation (21) gives the expression for photo-excitation from level $m$ to level $p_{k}$ in a radiation field with effective blackbody temperature $T_{\text {rad }}$ from a hot star underlying the X-ray line emitting plasma. As pointed out by Mewe $\&$ Schrijver (1978a) the radiation is diluted by a factor $W$ given by

$W=\frac{1}{2}\left[1-\left(1-\left(\frac{r_{*}}{r}\right)\right)^{1 / 2}\right]$,

where $r$ is the distance from the center of the stellar source of radius $r_{*}$. Close to the stellar surface the dilution factor $W=\frac{1}{2}$. For stars such as Capella or Procyon, we can take $W=\frac{1}{2}$, because the stellar surface which is the origin of the radiation irradiates coronal structures that are close to the stellar surface (Ness et al. 2001a). In a star such as Algol the radiation originates from another star, and $W$ is much lower (i.e. $W \simeq 0.01$, cf. Ness et al. 2001b), but due to the strong radiation field the radiation effect can still be important.

In their Table 8, Mewe \& Schrijver (1978a) give for information the radiation temperature for a solar photospheric field for $Z=6,7$, and 8 . In Table 3, we report the wavelengths at which the radiation temperature should be estimated for $Z=6,7,8,10,12,14$. These wavelengths correspond to the transitions between the ${ }^{3} \mathrm{~S}$ and ${ }^{3} \mathrm{P}$ levels $\left(\lambda_{f \rightarrow i}\right)$ and the ${ }^{1} \mathrm{~S}$ and ${ }^{1} \mathrm{P}$ levels $\left(\lambda_{6 \rightarrow r}\right)$.

The photo-excitation from the ${ }^{3} \mathrm{~S}$ level and ${ }^{3} \mathrm{P}$ levels is very important for low- $Z$ ions $\mathrm{Cv}, \mathrm{N}$ vi, O viI. For higher- $Z$ ions, this process is only important for very high radiation temperature ( $\sim$ few $10000 \mathrm{~K})$.

One can note that the photo-excitation between the levels ${ }^{1} \mathrm{~S}_{0}$ and ${ }^{1} \mathrm{P}_{1}$ is negligible compared to the photoexcitation between the ${ }^{3} \mathrm{~S}_{1}$ and ${ }^{3} \mathrm{P}_{0,1,2}$ levels. For example, 
Table 3. Wavelengths at which the radiation temperature $\left(T_{\text {rad }}\right)$ should be determined.

\begin{tabular}{ccccccc}
\hline & C V & N vi & O VII & Ne IX & Mg XI & Si XIII \\
$\lambda_{f \rightarrow i}(\AA)$ & 2280 & 1906 & 1637 & 1270 & 1033 & 864 \\
$\lambda_{6 \rightarrow r}(\AA)$ & 3542 & 2904 & 2454 & 1860 & 1475 & 1200 \\
\hline
\end{tabular}

for a very high value of $T_{\text {rad }}=30000 \mathrm{~K}$ the difference between the calculations taken or not taken into account the photo-excitation between ${ }^{1} \mathrm{~S}_{0}$ and ${ }^{1} \mathrm{P}_{1}$ is smaller than $20 \%$ for $\mathrm{C} \mathrm{V}$, where this effect is expected to be maximum.

\section{Results from extended calculations}

Using the above-mentioned atomic data, we have calculated the line intensity ratios $R$ and $G$ for $\mathrm{C}$ v, N vi, O viI, Ne IX, Mg XI, and Si XIII ${ }^{5}$ The wavelengths of these three (four) lines for each He-like ion treated in this paper are reported in Table 1 .

All the relevant processes detailed in Sects. 3 and 4 between the seven levels are taken into account (full resolution): radiative de-excitation, collisional electronic excitation and de-excitation, radiative and dielectronic recombination, photo-excitation and induced emission (between ${ }^{3} \mathrm{~S}_{1}$ and ${ }^{3} \mathrm{P}$ levels).

We considered a broad range of densities $\left(n_{\mathrm{e}}\right)$ and radiation temperatures $\left(T_{\text {rad }}\right)$ calculated for photo-excitation between the ${ }^{3} \mathrm{~S}$ level and the ${ }^{3} \mathrm{P}$ levels and a number of electron temperatures $\left(T_{\mathrm{e}}\right)$. As well, we considered different values of the dilution factor of the radiation field $(W)$ which could be used either for hot late-type stars or O, B stars.

We display the $G\left(T_{\mathrm{e}}\right)$ line intensity ratios, from Tables 4 to 9 , for the six ions, for five values of electron temperature $\left(T_{\mathrm{e}}\right)$ including the temperature of maximum line formation for the He-like lines (cf. Mewe et al. 1985); and for two or more values of the radiation temperature $\left(T_{\text {rad }}\right)$, and several values of $n_{\mathrm{e}}$. As one can note the ratio $G$ is as expected to be sensitive to $T_{\mathrm{e}}$, while it is almost insensitive to the exact values of $n_{\mathrm{e}}$ and $T_{\mathrm{rad}}$. The resonance line becomes sensitive at high density due to the depopulation of the $1 \mathrm{~s} 2 \mathrm{~s}^{1} \mathrm{~S}_{0}$ level to the $1 \mathrm{~s} 2 \mathrm{p}^{1} \mathrm{P}_{1}$ level (see Gabriel \& Jordan 1972). Since the sum $z+(x+y)$ is a constant or almost constant, the value of $G$ is independent of the exact value of the dilution factor $(W)$. Here the calculations were done for dilution factor $W=1 / 2$.

Finally, we display the $R\left(n_{\mathrm{e}}\right)$ line intensity ratios for the six ions, in Tables 10 to 69, for the same values of electron temperature $\left(T_{\mathrm{e}}\right)$ and much more values of radiation

\footnotetext{
${ }^{5}$ Tables 4 to 69 are only available in electronic form at the CDS via anonymous ftp to

cdsarc.u-strasbg.fr (130.79.128.5) or via

http://cdsweb.u-strasbg.fr/cgi-bin/qcat?J/A+A/376/1113
}

temperature $\left(T_{\mathrm{rad}}\right)$, and for three values of the dilution factor of the radiation field $W=0.5,0.1,0.01$.

Since as said previously in Sect. 4.3, the contribution of the blended dielectronic satellite lines depends on the spectral resolution, we give the values of $R$ and $G$ for $\mathrm{NeIX}, \mathrm{Mg}$ XI, and Si XIII, for four specific values of spectral resolutions $(F W H M)$ : RGS-1 at the first order (i.e. $\Delta \lambda=0.073,0.075$ and $0.078 \AA$ for Ne IX, Mg XI and Sixin respectively), LETGS (i.e. $\Delta \lambda=0.05 \AA$ ), HETGS-MEG (i.e. $\Delta \lambda=0.023 \AA$ ), and HETGS-HEG (i.e. $\Delta \lambda=0.012 \AA)$. In the cases where the differences $(<1 \%)$ are negligible between two or more spectral resolutions, we display the results together (e.g. RGS and LETGS).

\section{Conclusions}

For the first time, thanks to the new generation of X-ray satellites, Chandra and XMM-Newton, the diagnostics based on the line ratios of He-like ions could be used for powerful extra-solar plasma diagnostics (Warm Absorber in AGNs, stellar coronae, ...). These diagnostics are one of the keys for a better understanding of the solarstellar connection: heating of the coronae, magnetic activity, etc. In this work, we have calculated the line ratio $R$ and $G$ which allow, respectively, electron density and temperature diagnostics. These calculations are based partly on the previous work of Porquet \& Dubau (2000) and on different improvements of atomic data (see Sect. 4).

We have tabulated the results adapted for the different spectral resolutions of the spectrometers on board of Chandra and XMM-Newton for parameter ranges which correspond as much as possible to most cases of stellar coronae (hot-late type star, O B stars...).

Acknowledgements. The Space Research Organization Netherlands (SRON) is supported financially by NWO.

\section{Appendix A: Atomic data for the blended dielectronic satellite lines with $n=2,3,4$}

We report in this Appendix the atomic data related to the dielectronic satellite lines, calculated in this work for $Z=10,12$, and 14 , blended with one of their parent lines: forbidden, intercombination, and resonance.

The satellite line wavelengths and intensities have been obtained using a set of computer codes developed partly in University-College-London (SUPERSTRUCTURE: Eissner, Jones, Nussbaumer 1974) and in Meudon Observatory (AUTOLSJ: TFR group, Dubau J., Loulergue M. 1981). Multiconfigurational-wavefunctions are calculated in a "scaled" Thomas-Fermi-Dirac-Amaldi potentials, depending on linear scaling parameters $\left(\lambda_{\mathrm{s}}, \lambda_{\mathrm{p}}, \lambda_{\mathrm{d}} \ldots\right)$ different for $l$-orbitals, which are obtained through a self-consistent variational procedure 
on the energy sum of the first lowest (SL) terms. In SUPERSTRUCTURE, the level energies and the radiative probabilities are calculated in the relativistic Breit-Paul hamiltonian approach, which gives fine-structure bound and autoionizing levels. In the AUTOLSJ code, the autoionization probabilities are derived in the Distorted-wave approximation, using the same wavefunctions as in SUPERSTRUCTURE. For the present calculations, the following configuration were used: $1 \mathrm{~s}^{2} n l, 1 \mathrm{~s} 2 \mathrm{~s} n l$, and $1 \mathrm{~s} 2 \mathrm{p} n l$ for $n=2, \cdots, 5$ and $0 \leq l \leq n-1$.

The wavelengths of the dielectronic satellite lines calculated here should be compared to the "reference" wavelengths used in the Jacques Dubau's calculations respectively for NeIX, Mg XI, and Si XIII, $\lambda_{w}=13.4658,9.1740$, $6.6482 \AA, \lambda_{y}=13.5774,9.2395,6.6903 \AA, \lambda_{x}=13.5774$, $9.2358,6.6865 \AA, \lambda_{z}=13.7216,9.3219,6.7420 \AA$. One can notice that these wavelengths are not identical to the wavelengths of Vainshtein \& Safronova (1978) used in the calculation of the line ratios $R$ and $G$, tabulated in Table 1. Then in order to determine which dielectronic satellite lines are blended with one of the parent lines (forbidden, intercombination, and resonance), one should take into account the shift of the satellite line compared to the wavelengths chosen for the parent lines in the calculation of $R$ and $G$.

The values of $E_{\mathrm{s}}$, which is the energy of the satellite level $s$, used in this calculation are well reproduced using formula (29).

In Tables ${ }^{6}$ A.1, A.2, and A.3, the dielectronic satellite lines $n=2$, for $Z=10,12$, and 14 , respectively are reported. In Tables A.4, A.5, and A.6, the dielectronic satellite lines $n=3$, and 4 , for $Z=10,12$, and 14, respectively are reported.

\section{References}

Acton, L. W. 1978, ApJ, 225, 1069

Anders, E., \& Grevesse, N., 1989, Geochim. Cosmochim. Acta, 53, 197

Armour, I. A., Bashkin, S., Jelley, N. A., et al. 1979, J. Phys. Colloq. France, 40, C1-211

Armour, I. A., Silver, J. D., \& Träbert, E. 1981, J. Phys. B, 14,3563

Arnaud, M., \& Rothenflug, R. 1985, A\&AS, 60, 425

Arnaud, M., \& Raymond, J. 1992, ApJ, 398, 394

Audard, M., Behar, E., Güdel, M., et al. 2001, A\&A, 365, 329

Bely-Dubau, F., Gabriel, A. H., \& Volonté, S. 1979, MNRAS, 189,801

Blaha, M. 1971, Bull. Am. Astron. Soc., 3, 246 plus priv. comm.

Blumenthal, G. R., Drake, G. W. F., \& Tucker, W. H. 1972, ApJ, 172, 205

Brinkman, A. C., Gunsing, C. J. T., Kaastra, J. S., et al. 2000, ApJ, 530, L111

Canizares, C. R., Huenemoerder, D. P., Davis, D. S., et al. 2000, ApJ, 539, L41

${ }_{6}$ Tables A.1 to A.6 are only available in electronic form at the CDS.
Collinge, M. J., Brandt, W. N., Kaspi S., et al. 2001, ApJ, 557,2

Crespo López-Urrutia, J. R., Beiersdorfer, P., Savin, D. W., \& Widmann, K. 1998, Phys. Rev. A, 58, 238

den Herder, J. W., et al. 2001, A\&A, 365, L7

Doyle, J. G. 1980, A\&A, 87, 183

Eissner, W., Jones M., \& Nussbaumer, H. 1974, Comput. Phys. Commun., 8, 270

Engström, L., Jupén, C., Denne, B., et al. 1981, Phys. Scr., 22, 570

Gabriel, A. H., \& Jordan, C. 1969, MNRAS, 145, 241

Gabriel, A. H., \& Jordan, C. 1972, in Case studies in atomic collision physics, 2, 209, ed. McDaniel, \& McDowell

Hutton, R., Reistad, N., Engström, L., \& Huldt, S. 1985, Phys. Scr., 31, 506

Kaastra, J. S., \& Mewe, R. 1995, A\&A, 302, L13

Kaastra, J. S., Mewe, R., \& Nieuwenhuijzen, H. 1996, in UV and X-ray Spectroscopy of Astrophysical and Laboratory Plasmas, ed. K. Yamashita, \& T. Watanabe (Tokyo, Universal Academy Press, Inc.), 411 (SPEX)

Kaastra, J. S., Mewe, R., Liedahl, D. A., Komossa, S., \& Brinkman, A. C. 2000, A\&A, 354, L83

Kahn, S. M., Leutenegger, M. A., Cottam, J., et al. 2001, A\&A, 365, L312

Karim, K. R., \& Bhalla, C. P. 1992, Phys. Rev. A, 45, 3932

Karim, K. R. 1993, J. Phys. B: Atomic, Mol. Opt. Phys., 26, L725

Keenan, F. P., McCann, S. M., Kingston, A. E., \& McKenzie, D. L. 1987, ApJ, 318, 926

Liedahl, D. A. 1999, in X-ray Spectroscopy in Astrophysics, EADN School proceedings, 1997, ed. J. A. van Paradijs, \& J. A. M. Bleeker, 189

Lin, C. D., Johnson, W. R., \& Dalgarno, A. 1977, Phys. Rev. A, 15, 154

Mazzotta, P., Mazzitelli, G., Colafrancesco, S., \& Vittorio, N. 1998, A\&AS, 133, 403

McKenzie, D. L., \& Landecker, P. B. 1982, ApJ, 259, 372

Mewe, R. 1972, Solar Phys., 22, 114

Mewe, R., \& Schrijver, J. 1978a, A\&A, 65, 99

Mewe, R., \& Schrijver, J. 1978b, A\&A, 65, 115

Mewe, R., \& Schrijver, J. 1978c, A\&AS, 45, 11

Mewe, R., \& Gronenschild, E. H. B. M. 1981, A\&AS, 45, 11

Mewe, R., Gronenschild, E. H. B. M., \& van den Oord, G. H. J. 1985, A\&AS, 62, 197 (MEKAL)

Mewe, R., Kaastra, J. S., \& Liedahl, D. A. 1995a, Legacy, 6, 16 (MEKAL)

Mewe, R., Kaastra, J. S., Schrijver, C. J., van den Oord, G. H. J., \& Alkemade, F. J. M. 1995b, A\&A, 296, 477

Mewe, R. 1999, in X-ray Spectroscopy in Astrophysics, EADN School proceedings, 1997, ed. J. A. van Paradijs, \& J. A. M. Bleeker, 109

Mewe, R., Raassen, A. J. J., Drake, J. J., Kaastra, J. S., van der Meer, R. L. J., \& Porquet, D. 2001, A\&A, 368, 888

Neill, P. A., Träbert, E., Beiersdorfer, P., et al. 2000, Phys. Scr., 62, 141

Ness, J.-U., Mewe, R., Schmitt, J. H. M. M., et al. 2001a, A\&A, 367,282

Ness, J.-U., Schmitt, J. H. M. M., Burwitz, V., et al. 2001b, in preparation

Ogle, P. M., Marshall, H. L., Lee, J. C., \& Canizares, C. R. 2000, ApJ, 545, L81

Porquet, D., \& Dubau, J. 2000, A\&AS, 143, 495, Paper I 
Pradhan, A. K. 1982, ApJ, 263, 477

Pradhan, A. K. 1985, ApJ, 288, 824

Pradhan, A. K., \& Shull, J. M. 1981, ApJ, 249, 821

Sako, M., Kahn, S. M., Paerels, F., \& Liedahl, D. A. 2000, ApJ, 543, L115

Schmelz, J. T., Saba, J. L. R., Chauvin, J. C., \& Strong, K. T. 1997, ApJ, 477, 509

Schmidt, H. T., Forck, P., Grieser, M., et al. 1994, Phys. Rev. Lett., 72,1616

Schrijver, C. J., Mewe, R., van den Oord, G. H. J., \& Kaastra,
J. S. 1995, A\&A, 302, 438

Stefanelli, G. S., Beiersdorfer, P., Decaux, V., \& Widmann, K. 1995, Phys. Rev. A, 52, 3651

TFR group, Dubau, J., \& Loulergue, M. 1981, J. Phys. B, 15, 1907

Träbert, E., Beiersdorfer, P., Brown, G. V., et al. 1999, Phys. Rev. A, 60, 2034

Vainshtein, L. A., \& Safronova, U. I. 1978, Atom. Data Nucl. Data Tab., 21, 49

Zhang, H., \& Sampson, D. H. 1987, ApJS, 63, 487 